Background: CD5 is an antigen normally expressed on T-cells but can also be expressed in a small subset of B-cells and some non-Hodgkin B-cell lymphoproliferative disorders such as small lymphocytic lymphoma, marginal zone lymphoma and mantle cell lymphoma. When using a single immunohistochemical stain for each antigen on separate slides, it can be challenging to compare and correlate whether CD5 positivity is being seen in neoplastic B-cells or normal T-cells. This problem is amplified when the CD5 expressing B-cells are present in small numbers, such as in a bone marrow trephine.

Aim: In addition to the usual panel of immunohistochemical stains, we describe the application of two T-cell markers in a combined stain, CD5 (red, cytoplasmic) / CD3 (brown, cytoplasmic), to aid in the identification of CD5 co-expressing Bcells in small B-cell lymphoproliferative disorders.

Method: In a suspected small B-cell lymphoproliferative disorder, the red CD5 immunohistochemical stain is applied first and is positive in all normal T-cells and CD5-expressing B-cells. The brown CD3 stain is applied second, and also stains all the normal T-cells that express both CD3 and CD5, but does not stain any of the B-cells. As the darker brown stain is applied over the red, all CD3 and CD5 expressing T-cells that have been stained twice only appear brown, whereas the CD5 expressing Bcells appear red.

Conclusion: Following the application of these two T-cell markers to a single slide, it can be deduced that all brown staining cells are normal CD3 / CD5 expressing T-cells whereas the red staining cells are CD5-expressing B-cells.

\section{NEOPLASIA IN GASTRIC ADENOCARCINOMA AND PROXIMAL POLYPOSIS SYNDROME (GAPPS): GASTRIC RATHER THAN INTESTINAL PHENOTYPE}

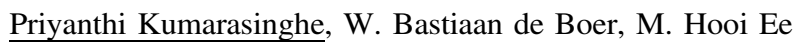
PathWest, WA

Background: Gastric adenocarcinoma and proximal polyposis of the stomach (GAPPS) was reported in 2011 as a new autosomal dominant gastric polyposis syndrome. It is characterised by exclusive involvement of the gastric body and fundus with sparing of the antrum by multiple, often 'carpet-like', polyps. These polyps have a morphologic spectrum that includes early microscopic lesions, 'fundic gland polyp' like lesions, dysplastic polyps and rare carcinomas. The neoplastic nature and progression have not been well characterised.

Aims: This study aims at a systematic and detailed morphological description of lesions in a cohort of GAPPS family members.

Design: Gastric biopsies and gastrectomies of 20 patients belonging to a previously defined GAPPS family were identified and their lesions were morphologically analysed in detail by 2 authors (BB,PK). When material was available, stains for MUC2, MUC5AC, MUC6, CDX2, Ki-67 and p53 were performed. Neoplastic lesions were categorised as low or high grade dysplasia, intestinal or gastric phenotype and invasive carcinoma.

Results: Dysplasia was identified in eight patients, either as a focal change within a non-adenomatous polyp (3) or as a gastric adenoma (3) or as both (2). All dysplastic lesions were gastric phenotype. Four were low grade and 4 were high grade dysplasia. One patient had carcinoma at presentation that was WHO tubular morphology and gastric phenotype. It was accompanied by fragments of dysplastic mucosa also of gastric phenotype showing 2 architectural patterns.

The spectrum of neoplastic lesions in GAPPS appears to be restricted to gastric phenotype irrespective of the type of lesions. Conclusions: The spectrum of gastric pathology associated with GAPPS is wider than previously reported and include newly identified hyper-proliferative aberrant pits, $\mathrm{PFH}$, predominantly gastric and/or hybrid dysplasia through to WHO tubular type adenocarcinoma.

\section{References}

1. Worthley DL, Phillips KD, Wayte N, et al. Gastric adenocarcinoma and proximal polyposis of the stomach (GAPPS): a new autosomal dominant syndrome. Gut 2012; 61: 774-9.

2. Yanaru-Fujisawa R, Nakamura S, Moriyama T, et al. Familial fundic gland polyposis, with gastric cancer. Gut 2012; 61: 1103-4.

3. Carneiro F, Hereditary gastric cancer. Pathologe 2012; 33: 231-4.

4. Omazzi B, Devani M, Tavani E, et al. Sporadic fundic gland polyps and proximal polyposis associated with gastric adenocarcinoma share a common antral $\mathrm{G}$ cell hyperplasia. Gut 2013; 62: 1088-9.

\section{THE ROLE OF FINE NEEDLE ASPIRATION CYTOLOGY IN THE ASSESSMENT OF LYMPHADENOPATHY}

Etty Hary Kusumastuti, Nila Kurniasari

Department of Pathology, Medical Faculty, Airlangga

University, Dr. Soetomo Hospital, Surabaya, Indonesia

Background: Fine needle aspiration cytology (FNAC) of lymph nodes is a simple, cost effective, out-patient procedure used for diagnosis of various cases of lymphadenopathies.

Aim: To evaluate the accuracy, sensitivity and specificity of the FNA-C as screening tool of lymphadenopathy.

Methods: FNA cytology archives of lymphadenopathies between January 2011 and December 2013 at Pathology Department Dr. Soetomo hospital was retrieved. Cytological and histological diagnostic were compared to estimate the accuracy of FNA-C. One hundred and eighteen cases were analyzed.

Result: The accuracy of FNA-C was $90.68 \%$, sensitivity was $89.52 \%$ and specificity was $100 \%$. Positive predictive value was $100 \%$, and negative predictive value was $54.17 \%$.

Conclusion: Cytological examination for lymphadenopathy by FNA-C is a high reliable tool, particularly in order to identify malignancy. However, for detailed subtyping of certain disease entities such as malignant lymphoma, surgical biopsy for histologic and immunohistochemical studies are required.

\section{CRIBRIFORM-MORULAR VARIANT OF PAPILLARY THYROID CARCINOMA: A STUDY OF THREE CASES FEATURING THE PIK3CA MUTATION}

Won-Jae Lee $^{1}$, Mi Jung Kwon ${ }^{2}$

${ }^{1}$ Department of Physical Medicine and Rehabilitation, Veterans Health Service Medical Center, Seoul, and ${ }^{2}$ Department of 
Pathology, Hallym University Sacred-Heart Hospital, Hallym University College of Medicine, Anyang, Korea

Background: The cribriform-morular variant of papillary thyroid carcinoma (CMV-PTC) is an unusual neoplasm with an important association with familial adenomatous polyposis in young women, characterized by a peculiar histology with mixed cribriform, papillary, solid, tall columnar, and morular patterns. However, it can also occur sporadically. The molecular pathogenesis of sporadic CMV-PTC is not completely understood. Here, we report cases of three patients with sporadic CMV-PTC with PIK3CA mutations.

Methods: Using sequencing analyses and immunohistochemistry, we examined KRAS, BRAF, PIK3CA, and CTNNB1 mutations and related proteins, including $\beta$-catenin, PTEN, CD10, estrogen receptor (ER), progesterone receptor (PR), cytokeratin 19, and cyclin D1 in three CMV-PTCs.

Results: The three patients were teen-aged women. The tumors were solitary and encapsulated without nodal metastasis. They showed no recurrence for more than six years following the operation. Three tumors were diffusely positive for $\beta$-catenin, cyclin D1, and PTEN. The biphasic immunohistochemical patterns between the morular and non-morular components were identified; the non-morular components were positive for ER, PR, and cytokeratin 19, whereas the morular components showed CD10 positivity. All tumors harbored the same mutation in exon 9, codon 545 of the PIK3CA gene (E545A), whereas the KRAS, BRAF, and CTNNB1 mutations were not detected.

Conclusions: This is the first study identifying the PIK3CA mutation specifically in sporadic CMV-PTC. The presence of the PIK3CA mutation and the wild-type KRAS, BRAF, CTNNB1 genes, and the intact PTEN expression in 3 sporadic CMV-PTCs may suggest the possible contribution of the PIK3CA mutation in its tumorigenesis.

\section{DIAGNOSTIC ROLE OF IMMUNOCYTOCHEMISTRY IN MALIGNANT PLEURAL MESOTHELIOMA}

Lam Nguyen Son ${ }^{1}$, Thanh Tran Dinh ${ }^{2}$, Dung Nguyen Huy ${ }^{3}$ ${ }^{1}$ Head of Pathology Department of Pham Ngoc Thach Hospital, Ho Chi Minh City, ${ }^{2} \mathrm{Head}$ of Oncology Department of Pham Ngoc Thach Hospital, and ${ }^{3}$ Director of Pham Ngoc Thach Hospital, Vietnam

Background: Malignant pleural mesothelioma is a difficult diagnosis, more severe cases may not perform by pleural biopsy. With these cases, the technique of immunocytochemistry was performed proposals.

Methods: A prospective, cross-sectional descriptive statistics with clinical series cases.

Results:

- Implementing 58 cases of malignant pleural mesothelioma by immunocytochemistry

- Technical Cytospin method has helped for immunocytochemistry have high results

- Should be done more staining with markers for the other diagnostic diseases: lung cancer and non-lung cancers that metastatic to pleura

- The results of the data:

- Sensitivity: $84.06 \%$

- Specificity: $100 \%$
- Positive Prognostic Value: $100 \%$

- Negative Prognostic Value: $92.25 \%$

- Accuracy $94.12 \%$

Conclusions: The diagnosis of malignant pleural mesothelioma is always a difficult diagnosis. Needing the differential diagnosis with other types of diseases, such as lung cancer and non-lung cancers that to pleural pulmonary metastases. Staining with immunocytochemistry in cases of pleural effusion showed relatively high value. This suggests that the ability to diagnose initially screening properties of this technique is very good, especially valuable in the diagnosis of severe disease progression cannot perform other diagnostic techniques.

References

1. Henderson D, Cagle P. Pleural Pathology. Pathology Int 2004; 54 (Suppl 1): S490-S505.

2. Metzgeroth G, Kuhn C, Schultheis B, et al. Diagnostic accuracy of cytology and immunocytology in carcinomatous effusions. Cytopathology 2008; 19: 205-11.

3. Motherby H, Nadjari B, Friegel P, et al. Diagnostic accuracy of effusion cytology. Diagn Cytopathol 1999; 20: 350-61.

4. Ikeda K, Tate G, Suzuki T, et al. Comparison of immunocytochemical sensitivity between formalin-fixed and alcoholfixed specimens reveals the diagnostic value of alcohol-fixed cytocentrifuged preparations in malignant effusion cytology. Am J Clin Pathol 2011; 136: 934-42.

5. Kouki Inai. Pathological Diagnosis of Asbestos-exposurerelated Diseases and Pathological Diagnosis of Mesothelioma. Training Course on Diagnosis of Asbestos Related Diseases, 2010: 7-10, 5-9.

\section{ADENOMATOID TUMOUR OF TUNICA ALBUGINEA OF TESTIS}

Tee Lang, Frank Lee

Khoo Teck Puat Hospital, Singapore

Background: Adenomatoid tumours are benign mesothelial neoplasm of paratesticular area and they commonly occur in the epipidymis. Uncommonly, adenomatoid tumours can arise from the tunica and present as intratesticular mass.

Aim: To describe a case of adenomatoid tumour presented as intratesticular tumour and to review its ultrasonographic appearance and histological features.

Method: A 39-year-old Sri Lankan man presented himself to the emergency department with acute onset of abdominal pain radiated to right groin. There was no obvious mass in the abdomen or groin. Serum tumour markers including $\beta$-HCG, $\alpha$ fetoprotein and lactate dehydrogenase were normal. An ultrasound scan identified a $10 \mathrm{~mm}$ hypoechoic solid nodule with minimal vascularity in the lower pole of right testis. The patient opted for conservative management but a follow- up ultrasound scan two months later showed a slight increased in size. An elective orchidectomy was performed to remove the nodule.

Result: An orchidectomy specimen with testis measured $40 \times 28 \times 20 \mathrm{~mm}$. The cut surface showed a firm yellow nodule at the lower pole measured $9 \times 5 \mathrm{~mm}$. The nodule had a well circumscribed border but lacked a capsule and it appeared to arise from the tunica albuginea abutted into the testicular parenchyma. Microscopically, the tumour showed an infiltrating growth pattern with cords and strands of oval tumour cells with eosinophilic cytoplasm within fibrous tissue. Occasional tumour 